\title{
Semiclassical Collapse of a Sphere of Dust
}

\author{
Roberto Casadio* and Giovanni Venturi ${ }^{\dagger}$ \\ Dipartimento di Fisica, Università di Bologna \\ and \\ Istituto Nazionale di Fisica Nucleare, Sezione di Bologna, Italy
}

June 21, 2021

\begin{abstract}
The semiclassical collapse of a homogeneous sphere of dust is studied. After identifying the independent dynamical variables, the system is canonically quantised and coupled equations describing matter (dust) and gravitation are obtained. The conditions for the validity of the adiabatic (Born-Oppenheimer) and semiclassical approximations are derived. Further on neglecting back-reaction effects, it is shown that in the vicinity of the horizon and inside the dust the Wightman function for a conformal scalar field coupled to a monopole emitter is thermal at the characteristic Hawking temperature.
\end{abstract}

*e-mail: Casadio@bologna.infn.it

${ }^{\dagger}$ e-mail: Armitage@bologna.infn.it 


\section{Introduction}

The canonical quantisation of general relativistic isotropic systems carried out in suitably chosen variables leads to the whole dynamics being determined by the Hamiltonian constraint [1] of the Arnowitt, Deser and Misner (ADM) construction [2]. Such an approach is particularly useful if one wishes to study the semiclassical regime of a system of self gravitating matter [3]. In this note we apply the ADM formalism to a model describing the collapse of a spherical object leading to a black hole. Further since one of the more intriguing aspects of quantum field theory in the curved background generated by a black hole is thermal Hawking radiation [4], we examine how it arises within such an approach.

In section 2 we briefly review the classical dynamics of an isotropic perfect fluid with zero pressure (dust) represented by a scalar field $\phi[5,6]$. In particular we observe that with a suitable gauge choice for the radial coordinate [6], the supermomentum constraint can be solved at the classical level and one is left with a Hamiltonian whose flow lines are the well known inhomogeneous Tolman solutions [7]. These reduce to the more specific Oppenheimer-Snyder model [8] if one also assumes homogeneity (subsection 2.1).

In contrast with a previous treatment [6] we do not impose the temporal gauge $\phi=-t$ which amounts to using the (classical) dust degree of freedom as a clock for the system and also restricts the solutions to the homogeneous case only. In fact we shall introduce time only after having quantised the remaining Hamiltonian constraint obtaining the WheelerDeWitt equation. This is not a trivial task and in subsection 2.1 we mention briefly the main conceptual problems one encounters: the operator ordering, the singularity at the origin and the absence of time.

In order to overcome all of these difficulties, in section 3 we illustrate a decomposition scheme for the Wheeler-DeWitt equation of the Oppenheimer-Snyder model in analogy with composite systems such as molecules which have two time (or mass) scales. The total wave function is factorised into a matter and a gravitational part [3] and on neglecting fluctuations (Born-Oppenheimer or adiabatic approximation), matter will follow gravity in the semiclassical limit for the latter.

Finally, in section 4 we introduce a massless scalar field conformally coupled to gravity and we allow it to interact locally with a monopole emitter localised near the horizon in coincidence with the last shell of the collapsing dust. This is done with the aim of obtaining information about the emitted radiation (temperature and intensity) as the last shell approaches the horizon. 
We use units for which $c=k_{\text {Boltzmann }}=1, \kappa \equiv 8 \pi G$, the Planck length is then $\ell_{p} \equiv \sqrt{\kappa \hbar}$ and the Planck mass is $m_{p}=\hbar / \ell_{p}$.

\section{The isotropic sphere of dust}

The most general three dimensional isotropic metric can be written as:

$$
{ }^{(3)} d s^{2}=e^{2 \nu} d \zeta^{2}+e^{2 \lambda} d \Omega^{2},
$$

where $d \Omega^{2}$ is the surface element of a unit 2-sphere, $\nu=\nu(\zeta, \sigma)$ and $\lambda=\lambda(\zeta, \sigma)$ are the gravitational degrees of freedom which depend only on the internal time variable $\sigma$ and the radial coordinate $\zeta$. A real massive scalar field $\phi=\phi(\zeta, \sigma)$ can be related to the dust 4velocity $U^{\alpha}$ through $U_{\alpha}=\phi_{, \alpha}$ and Hamilton's equations of motion for the self-gravitating sphere of dust can be obtained by variation of the action functional:

$$
S=4 \pi \int d \sigma d \zeta\left[\pi_{\nu} \frac{\partial \nu}{\partial \sigma}+\pi_{\lambda} \frac{\partial \lambda}{\partial \sigma}+\pi_{\phi} \frac{\partial \phi}{\partial \sigma}-N \mathcal{H}-N_{1} \mathcal{H}^{1}\right]
$$

with respect to $\nu, \lambda, \phi$, their conjugate momenta $\pi_{\nu}, \pi_{\lambda}$ and $\pi_{\phi}=-\sqrt{|g|} \mu U^{0}$. Here $\mu=\mu(\zeta, \sigma)$ is the rest mass density of the dust, $g$ is the determinant of the 4-metric whose pull-back on the 3-surface $\Sigma_{\sigma}$ of constant $\sigma$ is given in Eq. (2.1), $N$ and $N^{1}$ are the lapse and radial shift functions.

In the ADM formalism, $N$ and $N_{1}$ play the role of Lagrange multipliers for the Hamiltonian density $\mathcal{H}$ and the radial supermomentum $\mathcal{H}^{1}$ respectively, leading to the Hamiltonian and radial supermomentum constraints $[6,9]$ :

$$
\begin{aligned}
\mathcal{H}= & e^{-\nu-2 \lambda}\left[\frac{\kappa}{8} \pi_{\nu}^{2}-\frac{\kappa}{4} \pi_{\nu} \pi_{\lambda}+\frac{2}{\kappa} e^{4 \lambda}\left(2 \lambda^{\prime \prime}-2 \lambda^{\prime} \nu^{\prime}+3\left(\lambda^{\prime}\right)^{2}-e^{2(\nu-\lambda)}\right)\right] \\
& +\pi_{\phi}\left[1+e^{-2 \nu}\left(\phi^{\prime}\right)^{2}\right]^{1 / 2}=0 \\
\mathcal{H}^{1}= & -e^{-2 \nu}\left(\pi_{\nu}{ }^{\prime}-\nu^{\prime} \pi_{\nu}-\lambda^{\prime} \pi_{\lambda}\right)-e^{-2 \nu} \pi_{\phi} \phi^{\prime}=0,
\end{aligned}
$$

where a prime denotes the radial derivative $\partial / \partial \zeta$ and the above are secondary constraints in Dirac's language and arise because of the freedom of scaling the time variable $\sigma$ and the radial coordinate $\zeta$ on $\Sigma_{\sigma}$.

The residual gauge freedoms allow one to choose both the comoving gauge $U^{\alpha}=\delta_{0}^{\alpha}$, that is $\phi^{\prime}=0$ and then the scalar field is a function of time only, and the radial gauge $\lambda^{\prime} e^{\lambda-\nu}=\zeta$ which is possible if $\lambda^{\prime} e^{\lambda-\nu}$, considered as a new canonical variable, is a 
monotonic positive regular function of $\zeta$ at each value of $\sigma$. One then has $\lambda$ as the only gravitational degree of freedom and on solving the constraints Eqs. (2.3) and (2.4) one obtains a final Hamiltonian which does not contain any spatial derivatives so that the dynamics for different radii (shells) decouples. This is a consequence of the fact that dust is not subject to other interactions except gravity and Birkhoff's theorem ensures that each shell at constant $\zeta$ probes only the total mass in its interior [10].

After performing a further canonical transformation $r=e^{\lambda}$ from the Hamiltonian equations of motion one obtains a single equation:

$$
\dot{r}^{2}+2 r \ddot{r}+\left(1-\zeta^{2}\right)=0 .
$$

Here and in the following a dot will denote a derivative with respect to proper time $d \tau \equiv N d \sigma$. It is also useful to define $\rho \equiv \sqrt{\left(1-\zeta^{2}\right) / \epsilon}$, with:

$$
\epsilon= \begin{cases}+1 & 0 \leq \zeta<1 \\ 0 & \zeta=1 \\ -1 & \zeta>1\end{cases}
$$

In terms of this new radial coordinate the interior of the sphere of dust is given by $0 \leq \rho \leq$ $\rho_{0}$, where $\rho_{0}$ is the coordinate of the outer surface of the sphere: $0<\rho_{0}<1$ for $\epsilon=+1$, $\rho_{0}>0$ for $\epsilon=0,-1$. Of course, for $\epsilon=0$ the above definition is undefined (it corresponds to flat space) and we simply have $\zeta(\rho)=1$ for $0 \leq \rho \leq \rho_{0}$.

\subsection{The homogeneous case and matching with external Schwarzschild metric}

The solutions to Eq. (2.5) were obtained long ago [7] and, since the density $\kappa \mu(\rho, \tau)=$ $F^{\prime} /\left(r^{2} r^{\prime}\right)$ is determined only by the arbitrary function $F(\rho)$, we can restrict our analysis to the case in which the dust is homogeneous at each instant of proper time $\tau$ by taking $F(\rho)=2 \rho^{3} K_{0}$, where $K_{0}$ is a constant. The equations of geodesic motion then become:

$$
\begin{aligned}
r & =\rho K_{0} \partial_{\eta} h_{\epsilon}(\eta) \equiv \rho K_{c l}(\eta) \\
\tau & =\tau_{0} \pm K_{0} h_{\epsilon}(\eta)
\end{aligned}
$$

where $\tau_{0}$ is another constant and:

$$
h_{\epsilon}(\eta)= \begin{cases}\eta-\sin \eta & \epsilon=+1 \\ \eta^{3} / 6 & \epsilon=0 \\ \sinh \eta-\eta & \epsilon=-1,\end{cases}
$$


with $\eta$ a new time variable related to the proper time through $d \eta=\frac{\rho}{r} d \tau$. The density is now given by:

$$
\kappa \mu(\eta)=\frac{6 K_{0}}{K_{c l}^{3}(\eta)}
$$

and the line element by:

$$
d s^{2}=K_{c l}^{2}(\eta)\left[-d \eta^{2}+\frac{d \rho^{2}}{1-\epsilon \rho^{2}}+\rho^{2} d \Omega^{2}\right] .
$$

One then sees that the parameter $\eta$ is the conformal time and the portion of space-time filled with dust has the structure of a homogeneous Robertson-Walker manifold [10]. The case $\epsilon=+1$ corresponds to a sphere starting from its maximum radius $r=2 \rho K_{0}$ at $\eta=\pi$ which finally collapses to a point at $\eta=0$ while the other two cases $(\epsilon=0,-1)$ start from an initial radius at $\eta=\eta_{0}>0$ and again collapse at $\eta=0$.

We may now consider the exterior metric: the simplest assumption is to have vacuum outside the sphere of dust, so that isotropy and Birkhoff's theorem lead to the unique Schwarzschild solution [10] which must be matched at the surface $r_{0}$ of the dust sphere. This requires that each shell of dust move along a geodesic in both metrics $[10,11]$ implying that a shell at $\rho \leq \rho_{0}$ moves along $r=\rho K_{c l}(\eta) \leq r_{0}$ in a local metric of the Schwarzschild type with a mass parameter $M_{G}(\rho)=K_{0} \rho^{3} / \kappa$. The conformal time $\eta_{H}(\rho)$ at which each shell crosses its own Schwarzschild radius is then given by $r_{H}(\rho) \equiv 2 M(\rho)=\rho K_{c l}\left(\eta_{H}\right)$, so that from the point of view of a distant observer the first horizon to form is the outer one (and therefore the only relevant one) located at:

$$
r_{H} \equiv 2 M=2 K_{0} \rho_{0}^{3}
$$

which is the Schwarzschild radius of the sphere. This whole picture is also known as the Oppenheimer-Snyder model [8].

One may further express the interior conformal time $\eta$ as a function of the external Schwarzschild time $t$ for the geodesic trajectory at the surface $r=r_{0}$. This is rather complicated [10], but we are only interested in the asymptotic relations for $r_{0} \rightarrow r_{H}$. In such a limit one has:

$$
t \simeq \begin{cases}-2 M \ln \left\{\tan (\eta / 2)-\tan \left(\eta_{H} / 2\right)\right\} & \epsilon=+1 \\ -2 M \ln \left(\eta-\eta_{H}\right) & \epsilon=0 \\ -2 M \ln \left\{\tanh (\eta / 2)-\tanh \left(\eta_{H} / 2\right)\right\} & \epsilon=-1 .\end{cases}
$$


Eqs. (2.12) are of course singular at $\eta=\eta_{H}$ since we know that $t$ must diverge when the outer shell approaches the horizon, whereas nothing happens to a comoving observer. For all the above three cases the equations can be inverted to give:

$$
\eta-\eta_{H} \simeq C_{\epsilon} e^{-t / 2 M} \simeq C_{\epsilon}\left(1-\frac{2 M}{r_{0}(t)}\right)
$$

with $C_{\epsilon}$ a positive constant. The last factor is the square of the redshift for a photon escaping from near the black hole.

We may summarize our results as follows: the Einstein-Hilbert action for the metric (2.10) is given by:

$$
S_{G}=-\frac{V_{s}}{2 \kappa} \int d \tau\left[K \dot{K}^{2}-\epsilon K\right],
$$

where $V_{s}=4 \pi \int_{0}^{\rho_{0}} d \rho \rho^{2} / \sqrt{1-\epsilon \rho^{2}}$ is the spatial volume of the sphere and, in order to reproduce the solutions Eq. (2.7), the action for the dust must be:

$$
S_{M}=-\frac{V_{s}}{\kappa} K_{0} \int d \tau
$$

with a final Hamiltonian constraint:

$$
-\frac{1}{2}\left(\kappa \frac{\pi_{K}^{2}}{K}+\frac{\epsilon}{\kappa} K\right)+\frac{K_{0}}{\kappa}=0,
$$

Let us note that Eq. (2.16) has been obtained after having imposed the condition that matter satisfy the (classical) equation of state for dust (that is a perfect fluid with zero pressure) and after having solved for the classical radial momentum constraint Eq. (2.4). This in electrodynamics would correspond to a choice of gauge in order to eliminate unphysical degrees of freedom. On having identified the independent degrees of freedom one may proceed to quantise canonically Eq. (2.16) by replacing $\pi_{K} \mapsto \hat{\pi}_{K}=-i \hbar \frac{\partial}{\partial K}$. However one must also face the following issues:

1. Operator ordering. The formal mapping does not determine the quantum kinetic term uniquely:

$$
\frac{\pi_{K}^{2}}{K} \mapsto \frac{1}{\hat{K}^{a}} \hat{\pi}_{K}^{m} \frac{1}{\hat{K}^{b}} \hat{\pi}_{K}^{n} \frac{1}{\hat{K}^{c}}
$$

where $a, b, c, m$ and $n$ can be arbitrary integers such that $a+b+c=1$ and $m+n=2$ with $m$ and $n$ positive, since in all cases the same classical limit is obtained.

2. Singularity at the origin. The wave function $\Psi=\Psi(K, \phi)$ satisfying the quantised Wheeler-DeWitt equation must be zero at the origin $(K=0)$. 
3. Definition of time. Eq. (2.16) does not contain a time variable, so one must face the problem of the introduction of time in order to recover classical motion.

A consistent scheme solution to the above difficulties which also allows for the quantisation of matter is discussed in section 3 .

\section{The semiclassical collapse}

In the previous section we briefly reviewed for completeness the classical description of the evolution of a sphere of dust. We finally limited ourselves to the homogeneous case which we shall further analyse (a generalization to inhomogeneous collapse can be done by considering separately shells at different radii [6]).

As a guide for quantisation one has that in the semiclassical limit one must recover classical motion from some quantum state of gravity and matter. For our purpose it is convenient to consider a system consisting of a portion of a Robertson-Walker space-time plus a scalar field $\phi=\phi(\tau)$ whose total classical action is given by:

$$
\frac{S}{V_{s}}=\frac{1}{2} \int d \tau\left[-\frac{1}{\kappa}\left(K \dot{K}^{2}-\epsilon K\right)+K^{3}\left(\dot{\phi}^{2}-\mu^{2} \phi^{2}\right)\right],
$$

where $\mu=m / \hbar$ is the inverse of the Compton wavelength of the field $\phi$. On using the equations of motion for dust [5] and identifying the density as in Eq. (2.9) the above action becomes:

$$
\frac{S}{V_{s}}=-\frac{1}{2 \kappa} \int d \tau\left[K_{c l} \dot{K}_{c l}^{2}-\left(\epsilon K_{c l}-2 K_{0}\right)\right] \equiv \frac{S_{c l}}{V_{s}},
$$

where $K_{c l}$ is defined in Eq. (2.7). This is in agreement with what has been obtained in section 2: $S_{c l}=S_{G}+S_{M}$ where $S_{G}$ is given by Eq. (2.14) and $S_{M}$ by Eq. (2.15).

The Hamiltonian obtained from the action in Eq. (3.1) is:

$$
H=-\frac{1}{2}\left(\kappa \frac{\pi_{K}^{2}}{K}+\frac{\epsilon}{\kappa} K\right)+\frac{1}{2}\left(\frac{\pi_{\phi}^{2}}{K^{3}}+\mu^{2} \phi^{2} K^{3}\right) \equiv H_{G}+H_{M},
$$

where:

$$
\begin{aligned}
\pi_{K} & =-\frac{1}{\kappa} K \dot{K} \\
\pi_{\phi} & =K^{3} \dot{\phi} .
\end{aligned}
$$


Canonical quantisation $\left(\pi_{K} \rightarrow \hat{\pi}_{K}=-i \hbar \partial / \partial K, \pi_{\phi} \rightarrow \hat{\pi}_{\phi}=-i \hbar \partial / \partial \phi\right)$ then leads to the Wheeler-DeWitt equation:

$$
\frac{1}{2}\left[\kappa \hbar^{2} \frac{\partial_{K}^{2}}{K}-\frac{\epsilon}{\kappa} K-\frac{\hbar^{2}}{K^{3}} \frac{\partial^{2}}{\partial \phi^{2}}+\mu^{2} \phi^{2} K^{3}\right] \Psi(K, \phi)=0 .
$$

One may now choose an operator ordering in the gravitational kinetic term given by:

$$
\frac{\partial_{K}^{2}}{K} \equiv \frac{\partial^{2}}{\partial K^{2}} \frac{1}{K}
$$

and following a previously employed procedure [3] (analogous to that used in molecular dynamics) we express $\Psi$ in the factorized form:

$$
\Psi(K, \phi)=K \psi(K) \chi(\phi, K)
$$

which after multiplying on the L.H.S. of Eq. (3.5) by $\chi^{*}$ and integrating over the matter degrees of freedom leads to an equation for the gravitational part:

$$
\begin{aligned}
& \frac{1}{2}\left[\left(\kappa \hbar^{2} \frac{\partial^{2}}{\partial K^{2}}-\frac{\epsilon}{\kappa} K^{2}\right)+\frac{1}{\langle\tilde{\chi} \mid \tilde{\chi}\rangle}\left\langle\tilde{\chi}\left|\left(\frac{\hat{\pi}_{\phi}^{2}}{K^{2}}+\mu^{2} \phi^{2} K^{4}\right)\right| \tilde{\chi}\right\rangle\right] \tilde{\psi} \equiv \\
& \equiv\left[\hat{H}_{G} K+K\left\langle\hat{H}_{M}\right\rangle\right] \tilde{\psi}=-\frac{\kappa \hbar^{2}}{2} \frac{\left\langle\tilde{\chi}\left|\partial_{K}^{2}\right| \tilde{\chi}\right\rangle}{\langle\tilde{\chi} \mid \tilde{\chi}\rangle} \tilde{\psi} \equiv-\frac{\kappa \hbar^{2}}{2}\left\langle\partial_{K}^{2}\right\rangle \tilde{\psi},
\end{aligned}
$$

where we have defined a scalar product:

$$
\langle\chi \mid \chi\rangle \equiv \int d \phi \chi^{*}(\phi, K) \chi(\phi, K)
$$

Further we have set:

$$
\begin{aligned}
& \psi=\exp \left\{-i \int^{K} A\left(K^{\prime}\right) d K^{\prime}\right\} \tilde{\psi} \\
& \chi=\exp \left\{+i \int^{K} A\left(K^{\prime}\right) d K^{\prime}\right\} \tilde{\chi},
\end{aligned}
$$

with:

$$
A \equiv-i \frac{\left\langle\tilde{\chi}\left|\partial_{K}\right| \tilde{\chi}\right\rangle}{\langle\tilde{\chi} \mid \tilde{\chi}\rangle}
$$

If we multiply Eq. (3.8) by $\tilde{\chi}$ and subtract it from Eq. (3.5) we obtain:

$$
\tilde{\psi} K\left[\hat{H}_{M}-\left\langle\hat{H}_{M}\right\rangle\right] \tilde{\chi}+\kappa \hbar^{2}\left(\frac{\partial \tilde{\psi}}{\partial K}\right) \frac{\partial \tilde{\chi}}{\partial K}=\frac{\kappa \hbar^{2}}{2} \tilde{\psi}\left[\left\langle\frac{\partial^{2}}{\partial K^{2}}\right\rangle-\partial_{K}^{2}\right] \tilde{\chi}
$$

which is the equation for the matter (dust) wave function. Let us emphasize that by a suitable choice of operator ordering we have obtained a satisfactory form at the origin $(K=0)$ for the gravitational part $(K \tilde{\psi})$ of the wave function. 


\subsection{The dust wave function}

If we consider the semiclassical (WKB) approximation to the wave function $\tilde{\psi}$ one has:

$$
\frac{\partial \ln \tilde{\psi}}{\partial K} \simeq-\frac{i}{\hbar} \frac{\partial S_{e f f}}{\partial K}=-\frac{i}{\hbar} \pi_{K}
$$

where $S_{\text {eff }}$ is the effective action satisfying the Hamilton-Jacobi equation associated with the L.H.S. of Eq. (3.8), that is:

$$
S_{e f f}=-\frac{1}{2 \kappa} \int d \eta\left[K^{2} \dot{K}^{2}-K\left(\epsilon K-\left\langle\hat{H}_{M}\right\rangle\right)\right] .
$$

In such a semiclassical limit $\tilde{\psi}$ will be peaked at $K=K_{c}$ (classical trajectory). One may then define a (conformal) time variable:

$$
\frac{\partial}{\partial \eta} \equiv-i \hbar \kappa \frac{\partial \ln \tilde{\psi}}{\partial K} \frac{\partial}{\partial K}
$$

Further if the R.H.S. of Eq. (3.12) is small:

$$
\frac{\kappa \hbar^{2}}{2}\left[\left\langle\frac{\partial^{2}}{\partial K^{2}}\right\rangle-\partial_{K}^{2}\right] \tilde{\chi} \ll K_{c}\left\langle\hat{H}_{M}\right\rangle,
$$

one gets from Eq. (3.12) the Schrödinger equation for a harmonic oscillator:

$$
K_{c} \hat{H}_{M} \chi_{s}=i \hbar \frac{\partial \chi_{s}}{\partial \eta}
$$

where we have scaled the dynamical phase:

$$
\chi_{s} \equiv \tilde{\chi} \exp \left\{-\frac{i}{\hbar} \int^{\eta}\left\langle\hat{H}_{M}\left(\eta^{\prime}\right)\right\rangle K_{c} d \eta^{\prime}\right\}
$$

and omitted $\tilde{\psi}$ while setting $K=K_{c}$ which is where the semiclassical gravitational wave function is peaked and is a solution to the classical equation of motion obtained from Eq. (3.14). The solutions are [12]:

$$
\tilde{\chi}=\tilde{\chi}_{N}=N_{N} H_{N}(\gamma \phi) e^{-\gamma^{2} \phi^{2} / 2}=\left\langle\phi \mid \tilde{\chi}_{N}\right\rangle \text {, }
$$

where $H_{N}$ is the Hermite polynomial of degree $N, N_{N}$ a normalization factor and:

$$
\gamma^{2} \equiv \frac{\mu}{\hbar} K_{c}^{3}
$$

Further:

$$
K_{c}\left\langle\hat{H}_{M}\right\rangle_{N}=(N+1 / 2) \frac{\hbar^{2} \gamma^{2}}{K_{c}^{2}}=(N+1 / 2) \hbar \mu K_{c} \equiv(N+1 / 2) \hbar \omega_{c} .
$$


We may now consider the classical limit for the matter wave function. We introduce:

$$
\begin{aligned}
\chi_{c}(\phi, \eta)= & \sqrt{\frac{\pi^{1 / 2}}{\gamma}} \sum_{N} \frac{\left(\gamma \phi_{0}\right)^{N}}{\left(2^{N} N !\right)^{1 / 2}} e^{-\gamma^{2} \phi_{0}^{2} / 4} e^{-i(N+1 / 2) \omega_{c}\left(\eta-\eta_{0}\right)} \tilde{\chi}_{N}(\phi) \\
= & \sqrt{\frac{\pi^{1 / 2}}{\gamma}} \exp \left\{-\frac{1}{2} \gamma^{2}\left[\phi-\phi_{0} \cos \left(\omega_{c}\left(\eta-\eta_{0}\right)\right)\right]^{2}-i\left[\frac{1}{2} \omega_{c}\left(\eta-\eta_{0}\right)\right.\right. \\
& \left.\left.+\gamma^{2} \phi_{0} \phi_{i} \sin \left(\omega_{c}\left(\eta-\eta_{0}\right)\right)-\frac{1}{4} \gamma^{2} \phi_{0}^{2} \sin \left(2 \omega_{c}\left(\eta-\eta_{0}\right)\right)\right]\right\} .
\end{aligned}
$$

The superposition in Eq. (3.22) corresponds to a minimum wave packet displaced a distance $\phi_{0}$ at $\eta=\eta_{0}$ and oscillating about $\phi=0$ with a frequency $\omega_{c}$ which is constant in the adiabatic approximation. In the classical limit, $\hbar \rightarrow 0$, one has $\gamma \rightarrow \infty$ and the wave function Eq. (3.22) only has support on the classical trajectory:

$$
\left|\chi_{c l}(\phi, \eta)\right|^{2} \sim \delta\left(\phi-\phi_{c}(\eta)\right)
$$

with:

$$
\phi_{c}(\eta)=\phi_{0} \cos \left(\omega_{c}\left(\eta-\eta_{0}\right)\right)
$$

The coefficients in Eq. (3.22):

$$
\frac{\left(\gamma \phi_{0}\right)^{N}}{\left(2^{N} N !\right)^{1 / 2}} e^{-\gamma^{2} \phi_{0}^{2} / 4} \equiv \frac{\left(N_{c}\right)^{N}}{\sqrt{N !}} e^{-N_{c} / 2},
$$

have a maximum at $N=N_{c}=\gamma^{2} \phi_{0}^{2} / 2$ and their square obeys a Poisson distribution with average value $N_{c}$ and standard variation $\sqrt{N_{c}}$. Hence, if we just retain the term associated with the maximum in the dust wave function $\left(\chi_{c} \simeq \chi_{N_{c}}\right)$ one will have for the expectation value of the matter Hamiltonian for such a state:

$$
K_{c}\left\langle\hat{H}_{M}\right\rangle_{c}=\sum_{N=0}^{\infty} \frac{\left(N_{c}\right)^{2 N}}{N !} e^{-N_{c}}(N+1 / 2) \hbar \omega_{c} \simeq N_{c} \hbar \omega_{c},
$$

which is just the value of the classical Hamiltonian $K_{c} H_{M}$ for the oscillator in Eq. (3.24):

$$
\frac{1}{2}\left[K_{c}^{2}\left(\frac{\partial \phi_{c}}{\partial \eta}\right)^{2}+\mu^{2} K_{c}^{4} \phi_{c}^{2}\right]=\frac{1}{2} \gamma^{2} \phi_{0}^{2} \hbar \omega_{c}
$$

\subsection{The gravitational wave function}

The existence of the semiclassical limit implies that the gravitational wave function is peaked around some classical trajectory $K_{c}$ whose Hamilton-Jacobi action is given by $S_{\text {eff }}$ in Eq. (3.14). Since $\left\langle\hat{H}_{M}\right\rangle$ is independent of $K_{c}$ the trajectory will be given by one 
of the $K_{c l}$ defined in Eq. (2.7). This implies that $S_{\text {eff }}$ must be equal to $S_{c l}=S_{G}+S_{M}$ given in Eqs. (3.2). On comparing Eq. (3.14) with Eq. (3.2) above, one finds that in the L.H.S. of Eq. (3.8) one has:

$$
K_{c}\left\langle\hat{H}_{M}\right\rangle=K_{c l}\left\langle\hat{H}_{M}\right\rangle=\frac{1}{\kappa} K_{0} K_{c l}
$$

Moreover the R.H.S. of Eq. (3.8) must be negligible:

$$
-\frac{\kappa \hbar^{2}}{2}\left\langle\frac{\partial^{2}}{\partial K^{2}}\right\rangle \ll K_{c l}\left\langle\hat{H}_{M}\right\rangle .
$$

Eq. (3.28) means that the mean matter Hamiltonian behaves as dust upon neglecting fluctuations (Eq. (3.29) and the R.H.S. of Eq. (3.12)) and matter follows gravitation adiabatically (Born-Oppenheimer approximation).

It is straightforward to derive an expression for $\tilde{\psi}$ which satisfies Eq. (3.8) in the semiclassical limit and neglecting fluctuations. Suppose that at time $\eta_{0}$ for which $\partial_{\eta} K_{c l}=0$ (that is $\eta_{0}=0$ or $\pi$ for $\epsilon=+1$ and $\eta_{0}=0$ for $\epsilon=0,-1$ ) the gravitational wave function is a gaussian packet centred at the value $K_{c l}\left(\eta_{0}\right)$ with width $b$ :

$$
\tilde{\psi}\left(K, \eta_{0}\right)=\exp \left\{-\frac{\left(K-K_{c l}\left(\eta_{0}\right)\right)^{2}}{2 b^{2}}\right\},
$$

where for simplicity we have omitted a normalization factor. At a succeeding time $\eta \tilde{\psi}$ will be given by:

$$
\tilde{\psi}(K, \eta)=\int d K^{\prime} G_{\epsilon}\left(K-\epsilon K_{0}, \eta ; K^{\prime}-\epsilon K_{0}, \eta_{0}\right) \tilde{\psi}\left(K^{\prime} ; \eta_{0}\right)
$$

where $G_{\epsilon}$ is the Green's function for the (inverted) harmonic oscillator for $\epsilon=+1(-1)$ or for a particle moving in a linear potential for $\epsilon=0$ [13]. One then obtains:

$$
\begin{aligned}
\tilde{\psi}(K, \eta)= & \exp \left\{i \frac{\left(K-\epsilon K_{0}\right)^{2}}{2 \hbar \kappa\left(\partial_{\eta}^{2} h_{\epsilon}\right)}\left(1-\alpha^{2} b^{2}\right) \sqrt{1-\epsilon\left(\partial_{\eta}^{2} h_{\epsilon}\right)^{2}}\right\} \\
& \times \exp \left\{i \frac{\alpha^{2} \hbar \kappa}{2 b^{2}} K_{0}\left(\partial_{\eta}^{2} h_{\epsilon}\right)\left(K_{0} \sqrt{1-\epsilon\left(\partial_{\eta}^{2} h_{\epsilon}\right)^{2}}+2 \epsilon\left(K-\epsilon K_{0}\right)\right)\right\} \\
& \times \exp \left\{-\frac{\alpha^{2}}{2}\left(K-K_{c l}(\eta)\right)^{2}\right\},
\end{aligned}
$$

where $h_{\epsilon}(\eta)$ has been defined in Eq. (2.8) and:

$$
\alpha=\frac{b}{\left[\kappa^{2} \hbar^{2}\left(\partial_{\eta}^{2} h_{\epsilon}\right)^{2}+b^{4}\left(1-\epsilon\left(\partial_{\eta}^{2} h_{\epsilon}\right)^{2}\right)\right]^{1 / 2}} .
$$


From Eq. (3.32) one immediately obtains:

$$
\frac{\partial \ln \tilde{\psi}}{\partial K}=i \frac{K_{0}}{\hbar \kappa} \partial_{\eta}^{2} h_{\epsilon}(\eta)+O\left(\hbar^{0}\right)=-\frac{i}{\hbar} \pi_{K}+O\left(\hbar^{0}\right)
$$

in agreement with Eq. (3.13) to leading order in $\hbar$. We further note that the total gravitational wave function is $K \tilde{\psi}(K)$ which is 0 for $K=0$ as desired: this solution is a consequence of our choice of ordering, which however is not relevant in the semiclassical limit.

The absolute value of the gravitational wave function is:

$$
|\tilde{\psi}(K, \eta)|^{2}=\exp \left\{-\alpha^{2}\left(K-K_{c l}(\eta)\right)^{2}\right\},
$$

If one now considers the limit $\hbar \rightarrow 0$ followed by $b \rightarrow 0$ (the classical point-like limit) the classical trajectory is obtained:

$$
\alpha \rightarrow \infty \quad \Longrightarrow \quad \frac{K^{2}|\tilde{\psi}|^{2}}{\left\langle\tilde{\psi}\left|\hat{K}^{2}\right| \tilde{\psi}\right\rangle} \rightarrow \delta\left(K-K_{c l}\right)
$$

where $\left\langle\tilde{\psi}\left|\hat{K}^{2}\right| \tilde{\psi}\right\rangle$ is the norm of the complete wave function $K \tilde{\psi}$ and is given in Appendix A. With the above limits interchanged, that is $b \rightarrow 0$ with $\hbar$ finite, one would obtain:

$$
\alpha \rightarrow 0 \Longrightarrow \frac{K^{2}|\tilde{\psi}|^{2}}{\left\langle\tilde{\psi}\left|\hat{K}^{2}\right| \tilde{\psi}\right\rangle} \rightarrow 0
$$

However, we expect that when $b$ becomes smaller than the Planck length $\ell_{p}$ quantum gravitational effects (fluctuations) become significant. Hence it is more sensible to consider the limit for which one has $b \sim \ell_{p}$ (corresponding to a minimum size wave packet of the order of the Planck length) and then consider $\ell_{p} \rightarrow 0$. For such a case:

$$
\alpha \sim \ell_{p}^{-1} \rightarrow \infty
$$

which again leads to Eq. (3.36).

If we also consider the semiclassical limit for matter from Eqs. (3.22) and (3.28) one has:

$$
\frac{1}{\kappa} K_{0} K_{c l}=N_{c} \hbar \mu K_{c l}
$$

and, taking into account the junction condition Eq. (2.11):

$$
M_{G}=\rho_{0}^{3} N_{c} \hbar \mu
$$

We may then conclude that the total mass $M_{G}$ of the collapsing sphere is proportional to the number (large) of dust quanta $N_{c}$. The total euclidean action $S_{e}$ (the gravitational 
entropy) of the system once the black hole has formed is known to be proportional to the area of the horizon [14]. On using our result Eq. (3.40) it also follows that:

$$
S_{e}=\frac{A}{4}=4 \pi M^{2}=4 \pi\left(\rho_{0}^{3} \ell_{p}^{2} N_{c} \mu\right)^{2},
$$

which implies that $S_{e}$ is also quantised in terms of the dust quanta.

The above apparently differ from the result obtained previously, $M_{G} \sim m_{p} \sqrt{n}$ [15], but we observe that the $N_{c}$ appearing in Eq. (3.40) are energy quantum numbers of the dust, while the $n$ in Bekenstein's formula comes from quantising the gravitational Hamiltonian. In fact, let us consider the case $\epsilon=+1$. Eq. (3.8), using Eqs. (3.28) and (3.29), becomes:

$$
\frac{1}{2}\left[-\kappa \hbar^{2} \frac{\partial^{2}}{\partial K^{2}}+\frac{K}{\kappa}\left(K-2 K_{0}\right)\right] \tilde{\psi}=0
$$

which may be re-written as [16]:

$$
\frac{1}{2}\left[\kappa \hbar^{2} \frac{\partial^{2}}{\partial X_{n}^{2}}+\frac{1}{\kappa} X_{n}^{2}\right] \tilde{\psi}=\frac{K_{0, n}^{2}}{2 \kappa} \tilde{\psi}
$$

where $X_{n} \equiv K-K_{0, n}$. The solutions are:

$$
\tilde{\psi}=H_{n}\left(X_{n} / \ell_{p}\right) e^{-X_{n}{ }^{2} / 2 \ell_{p}}
$$

with $H_{n}$ the Hermite polynomial of degree $n$ and $K_{0, n}{ }^{2}=\ell_{p}^{2}(n+1 / 2)$. It then follows that:

$$
M_{G}=m_{p} \rho_{0}^{3} \sqrt{n+1 / 2},
$$

which is essentially Bekenstein's formula. Let us note that in the other two cases $\epsilon=0,-1$ no analogous discrete quantisation exists.

\subsection{Consistency conditions}

Let us first see how quantisation modifies the geodesic motion since, even if $\tilde{\psi}(K, \eta)$ is peaked on the classical value $K_{c l}$, one has fluctuations around it. Ehrenfest's theorem [17] (with the classical limit) will be satisfied if the average position $\langle\hat{r}\rangle$ will coincide with the classical trajectory up to small fluctuations. That is:

$$
\rho_{0} \frac{\left\langle\tilde{\psi}\left|\hat{K}^{3}\right| \tilde{\psi}\right\rangle}{\left\langle\tilde{\psi}\left|\hat{K}^{2}\right| \tilde{\psi}\right\rangle} \equiv \rho_{0}\langle\hat{K}\rangle=r_{0}(\eta)+O(\hbar) .
$$

What one actually obtains is (see Appendix A for the details):

$$
\langle\hat{K}\rangle=\frac{\sqrt{\pi} \alpha K_{c l}\left(3+2 \alpha^{2} K_{c l}^{2}\right) \operatorname{Erfc}\left(-\alpha K_{c l}\right)+2\left(1+\alpha^{2} K_{c l}^{2}\right) e^{-\alpha^{2} K_{c l}{ }^{2}}}{\alpha\left[\sqrt{\pi}\left(1+2 \alpha^{2} K_{c l}^{2}\right) \operatorname{Erfc}\left(-\alpha K_{c l}\right)+2 \alpha K_{c l} e^{-\alpha^{2} K_{c l}{ }^{2}}\right]},
$$


and in the classical point-like limit, Eq. (3.36) or Eq. (3.38), this expression gives exactly the classical trajectory:

$$
\rho_{0}\langle\hat{K}\rangle \rightarrow \rho_{0} K_{c l}
$$

Ehrenfest's theorem, as illustrated in Eq. (3.46), implies that fluctuations are negligible. In our case this means that one must have:

$$
\Delta \equiv \frac{\left|\left\langle\hat{K}^{2}\right\rangle-\langle\hat{K}\rangle^{2}\right|}{\langle\hat{K}\rangle} \ll\langle\hat{K}\rangle .
$$

For the wave function $\tilde{\psi}$ one finds (see Appendix A):

$$
\begin{gathered}
\left\langle\hat{K}^{2}\right\rangle \equiv \frac{\left\langle\tilde{\psi}\left|\hat{K}^{4}\right| \tilde{\psi}\right\rangle}{\left\langle\tilde{\psi}\left|\hat{K}^{2}\right| \tilde{\psi}\right\rangle}= \\
\frac{\sqrt{\pi}\left(3+12 \alpha^{2} K_{c l}^{2}+4 \alpha^{4} K_{c l}^{4}\right) \operatorname{Erfc}\left(-\alpha K_{c l}\right)+2 \alpha K_{c l}\left(5+2 \alpha^{2} K_{c l}^{2}\right) e^{-\alpha^{2} K_{c l}{ }^{2}}}{2 \alpha^{2}\left[\sqrt{\pi}\left(1+2 \alpha^{2} K_{c l}^{2}\right) \operatorname{Erfc}\left(-\alpha K_{c l}\right)+2 \alpha K_{c l} e^{\left.-\alpha^{2} K_{c l}{ }^{2}\right]}\right.},
\end{gathered}
$$

so that:

$$
\frac{\Delta}{\langle\hat{K}\rangle} \sim \frac{1}{\alpha^{2}} \rightarrow 0
$$

in the classical point-like limit, Eq. (3.36) or Eq. (3.38). However if one considers the limiting procedure used in Eq. (3.37) one has:

$$
\frac{\Delta}{\langle\hat{K}\rangle} \rightarrow \frac{3 \pi}{8}-1 \simeq 0.18
$$

which implies Eq. (3.46) is not satisfied.

It is also interesting to consider small black holes. Let us then take $K_{c l}$ small at fixed $\hbar$ and $b \sim \ell_{p}$, one then has:

$$
\Delta \sim \frac{3 \pi-8}{4 \sqrt{\pi}} \frac{1}{\alpha} \sim \ell_{p}
$$

which of course implies that one must have $r_{0}(\eta)=\rho_{0} K_{c l}(\eta) \gg \ell_{p}$. Since we are only interested in the limit for which $r_{0}$ approaches $r_{H}$ from outside, one must have $2 M \gg$ $\Delta \sim \ell_{p}$ or:

$$
M_{G} \gg m_{p}
$$

This coincides with what one would expect from naive quantum mechanical considerations. Indeed, if $\lambda_{b h}=\kappa \hbar / M$ is the Compton wave length of the black hole, it should be much less than its Schwarzschild radius $2 M$ for the semiclassical approximation to work. This corresponds exactly to Eq. (3.54). 
The remaining consistency conditions are Eqs. (3.29) and (3.16) to which one may add the condition for the validity of the adiabatic approximation, that is that the time dependence in the matter wave function induced by the slow variable (gravitation) be small. This essentially requires that in Eq. (3.12) the second term on the L.H.S. be larger than the third (evaluated using Eq. (3.13)). On using the the solutions Eq. (3.19) for $N=N_{c}$ which will approximate classical dust (analogous results are obtained for the "vacuum" state $N=0$ for matter) into the R.H.S. of Eq. (3.8) one obtains (see Appendix B for more details):

$$
\frac{\kappa \hbar^{2}}{2}\left\langle\frac{\partial^{2}}{\partial K^{2}}\right\rangle \simeq \frac{9 \kappa \hbar^{2}}{16 K_{c l}^{2}}\left(N_{c}^{2}+N_{c}+1\right) \leq \frac{9 \kappa \hbar^{2} \rho_{0}^{2}}{64 M^{2}}\left(N_{c}^{2}+N_{c}+1\right),
$$

where $K_{c l}$ is evaluated at the minimum value we need, that is $r_{H} / \rho_{0}=2 \mathrm{M} / \rho_{0}$. The condition Eq. (3.29) then becomes:

$$
\begin{aligned}
\frac{\kappa \hbar^{2}}{2} \frac{\left\langle\partial_{K}^{2}\right\rangle}{K_{c l}\left\langle\hat{H}_{M}\right\rangle} & \simeq \frac{9 \kappa \hbar}{8 \mu K_{c l}^{3}} \frac{N_{c}^{2}+N_{c}+1}{2 N_{c}+1} \\
& \leq \frac{9 \kappa \hbar \rho_{0}^{3}}{64 \mu M^{3}} \frac{N_{c}^{2}+N_{c}+1}{2 N_{c}+1} \\
& \ll 1,
\end{aligned}
$$

Analogously, Eq. (3.16) yields:

$$
\begin{aligned}
\frac{\kappa \hbar^{2}}{2} \frac{\left|\left[\left\langle\partial_{K}^{2}\right\rangle-\partial_{K}^{2}\right] \tilde{\chi}\right|}{K_{c l}\left\langle\hat{H}_{M}\right\rangle} & \simeq \frac{\kappa \hbar}{\mu K_{c l}^{3}} \frac{N_{c}^{2}+N_{c}+1}{2 N_{c}+1} \\
& \leq \frac{\kappa \hbar \rho_{o}^{3}}{8 \mu M^{3}} \frac{N_{c}^{2}+N_{c}+1}{2 N_{c}+1} \\
& \ll 1,
\end{aligned}
$$

which is essentially the same as Eq. (3.56).

Finally from Eqs. (3.12) and (3.15) one must have:

$$
\begin{aligned}
\hbar \frac{\partial K_{c l}}{\partial \eta} \frac{\left|\left\langle\overleftarrow{\partial_{K}} \partial_{K}\right\rangle\right|^{1 / 2}}{K_{c l}\left\langle\hat{H}_{M}\right\rangle} & \simeq \frac{\partial K_{c l}}{\partial \eta} \frac{\sqrt{N_{c}^{2}+N_{c}+1}}{\mu K_{c l}^{2}\left(2 N_{c}+1\right)} \\
& \leq \frac{3}{2 \sqrt{2}} \frac{1}{\mu^{2} M} \frac{\sqrt{N_{c}^{2}+N_{c}+1}}{\left(2 N_{c}+1\right)} \times \begin{cases}\sqrt{1-\rho_{0}^{2}} & \epsilon=+1 \\
1 & \epsilon=0 \\
\sqrt{1+\rho_{0}^{2}} & \epsilon=-1\end{cases} \\
& \ll 1,
\end{aligned}
$$

which, for the periodic case $\epsilon=+1$, can be understood as the ratio between the periods of the gravitational motion and the one for the matter state $\chi_{N_{c}}$; the fact it is small is 
a statement of the adiabatic approximation. Since for the semiclassical wave packet in Eq. (3.22) $N_{c} \gg 1$, the above three conditions are essentially the same and, on using Eq. (3.40) to express $N_{c}$ as a function of $M$, one obtains:

$$
M \gg \frac{1}{\mu}
$$

which means that the Schwarzschild radius of the dust must be much larger than the Compton wave length of the dust particles, or:

$$
M_{G} \gg \frac{m_{p}^{2}}{m}
$$

Since $m \ll m_{p}$ for all known elementary particles, the latter expression is a stronger condition than Eq. (3.54). The fact that the adiabatic approximation is not valid if Eq. (3.59) is not satisfied is not surprising. Indeed in such a case fluctuations, corresponding to the creation of matter particles, are large (and the evaporation time small [4]).

\section{Coupling with a conformal scalar field}

An observer modelled by a point-like monopole detector localized at a fixed $r>r_{H}$ and coupled to the radiation field can be used to reveal Hawking radiation [4]. Since the detector trajectory approaches the uniformly accelerated one in Minkowski space-time as $r \rightarrow r_{H}$, one may take the radiation field in the Unruh vacuum and proceed as in the calculation of the Unruh effect [19]. One then obtains that the observer detects a thermal flux with a temperature given by:

$$
T_{H}=\frac{\hbar}{8 \pi M}
$$

For the flat case (Minkowski space-time) one can also consider a quantum detector described by a gaussian wave packet and one gets the usual Unruh effect for a classical point-like limit as in Eq. (3.36) [20].

Here we shall examine a different approach. Let us consider the outer dust shell of the sphere situated at $\rho=\rho_{0}$ as a quantum system characterized by an internal energy spectrum which is related to the number of dust quanta in the shell itself. From the point of view of a distant observer the position of the shell is not a classical variable but is a quantum observable determined by the gravitational wave function describing the semiclassical collapse obtained in the previous section. 
We consider an isotropic massless scalar field $\varphi=\varphi(\rho, \eta)$ conformally coupled to gravity [18] and to the outer shell of dust. Its Lagrangian density will be given by:

$$
\begin{aligned}
\mathcal{L}_{\varphi}= & -\frac{1}{2}\left[\partial_{\mu} \varphi \partial^{\mu} \varphi+\frac{1}{6} R \varphi^{2}\right] \\
& +\int d \tau \int d \rho \int_{0}^{+\infty} d K \frac{|K \tilde{\psi}(K, \tau)|^{2}}{\left\langle\tilde{\psi}\left|\hat{K}^{2}\right| \tilde{\psi}\right\rangle} \delta\left(\rho-\rho_{0}\right) Q(\rho, \tau) \varphi(\rho, \tau),
\end{aligned}
$$

where $R$ is the curvature scalar, $Q(\rho, \tau)$ describes a particle (monopole) emitter and the factor $\delta\left(\rho-\rho_{0}\right)|K \tilde{\psi}|^{2} /\left\langle\tilde{\psi}\left|\hat{K}^{2}\right| \tilde{\psi}\right\rangle$ forces the interaction to be localized on the outer shell. In particular, in the classical point-like limit (Eq. (3.36)) it leads to $\delta\left(\rho-\rho_{0}\right) \delta\left(K-K_{c l}\right)=$ $\delta\left(r-r_{0}\right)$. The reason we consider an excited monopole (emitter) is to avoid the problem of back-reaction on matter and gravity. Further in our case the observer is distant from the dust sphere and static, this is the reason we shall use Schwarzschild coordinates for it.

Let us assume the emitter has a discrete set of internal energy eigenstates described by $|E\rangle$ where $E$ is the energy observed by our distant observer. We suppose the emitter is initially in an excited state $E_{0}$ and decays by emitting quanta of the scalar field $\varphi$ to a state $E_{0}-\hbar \omega$. One may then estimate, using first order perturbation theory, the total probability amplitude $P(\omega, \bar{\eta})$ for the emitter to decay in a finite conformal time. It will be given by:

$$
\begin{aligned}
P(\omega, \bar{\eta}) & =\frac{Q_{\omega}^{2}}{\hbar^{2}} \int_{\tau\left(\eta_{0}\right)}^{\tau(\bar{\eta})} d \tau^{\prime \prime} \int_{\tau\left(\eta_{0}\right)}^{\tau(\bar{\eta})} d \tau^{\prime} e^{i \omega\left(t^{\prime \prime}-t^{\prime}\right)} D_{\epsilon}^{+}\left(\eta^{\prime \prime}, \eta^{\prime}\right) \\
& =\frac{Q_{\omega}^{2}}{\hbar^{2}} \int_{\eta_{0}}^{\bar{\eta}} K_{c l}\left(\eta^{\prime \prime}\right) d \eta^{\prime \prime} \int_{\eta_{0}}^{\bar{\eta}} K_{c l}\left(\eta^{\prime}\right) d \eta^{\prime} e^{i \omega\left(t^{\prime \prime}-t^{\prime}\right)} D_{\epsilon}^{+}\left(\eta^{\prime \prime}, \eta^{\prime}\right)
\end{aligned}
$$

where $Q_{\omega} \equiv\left|\left\langle E_{0}\left|Q\left(\rho_{0}, 0\right)\right| E_{0}-\hbar \omega\right\rangle\right|$ is the absolute value of the matrix element of the monopole between the states with energy $E_{0}$ and $E_{0}-\hbar \omega, t^{\prime \prime}=t\left(\eta^{\prime \prime}\right)$ and $t^{\prime}=t\left(\eta^{\prime}\right)$ are the Schwarzschild times measured by the distant observer expressed in term of the conformal times as given in Eq. (2.12), and $\bar{\eta}$ is an upper cut off such that $\eta_{H}<\bar{\eta}<\eta_{0}$. Further during the interval $\left(\eta_{0}, \bar{\eta}\right)$ the static monopole emitter near the horizon is immersed in the gravitational wave packet associated with the last dust shell and $D_{\epsilon}^{+}$is the Wightman function [18] for the isotropic conformal scalar field in the metric Eq. (2.10) evaluated at the same spatial point $\rho^{\prime \prime}=\rho^{\prime}=\rho$, but with the $K_{c l}$ in the denominator "smeared" by the wave function $K \tilde{\psi}$ of Eq. (3.32):

$$
\begin{aligned}
D_{\epsilon}^{+}\left(\eta^{\prime \prime}, \eta^{\prime}\right) \equiv & \int_{0}^{+\infty} d K^{\prime \prime} \frac{\left|K^{\prime \prime} \tilde{\psi}\left(K^{\prime \prime}, \eta^{\prime \prime}\right)\right|^{2}}{\left\langle\tilde{\psi}\left(\eta^{\prime \prime}\right)\left|\hat{K}^{2}\right| \tilde{\psi}\left(\eta^{\prime \prime}\right)\right\rangle} \\
& \times \int_{0}^{+\infty} d K^{\prime} \frac{\left|K^{\prime} \tilde{\psi}\left(K^{\prime}, \eta^{\prime}\right)\right|^{2}}{\left\langle\tilde{\psi}\left(\eta^{\prime}\right)\left|\hat{K}^{2}\right| \tilde{\psi}\left(\eta^{\prime}\right)\right\rangle} \frac{D_{\epsilon}\left(\eta^{\prime \prime}, \eta^{\prime}\right)}{K^{\prime \prime} K^{\prime}}
\end{aligned}
$$


where:

$$
D_{\epsilon}\left(\eta^{\prime \prime}, \eta^{\prime}\right)= \begin{cases}\frac{\hbar}{8 \pi^{2}\left[\cos \left(\eta^{\prime \prime}-\eta^{\prime}-i \varepsilon\right)-1\right]} & \epsilon=+1 \\ -\frac{\hbar}{4 \pi^{2}\left(\eta^{\prime \prime}-\eta^{\prime}-i \varepsilon\right)^{2}} & \epsilon=0,-1 .\end{cases}
$$

In using these propagators one is limiting oneself to $s$-waves. However this is known not to be too restrictive since there is a potential barrier outside the horizon in the Klein-Gordon equation for the scalar field which, in practice, suppresses all higher angular momentum modes. On performing the $K^{\prime \prime}, K^{\prime}$ integrations one finds:

$$
K_{c l}\left(\eta^{\prime \prime}\right) K_{c l}\left(\eta^{\prime}\right) D_{\epsilon}^{+}\left(\eta^{\prime \prime}, \eta^{\prime}\right)=R\left(\eta^{\prime \prime}\right) R\left(\eta^{\prime}\right) D_{\epsilon}\left(\eta^{\prime \prime}, \eta^{\prime}\right)
$$

where (see Appendix A):

$$
\begin{aligned}
R(\eta) & \equiv K_{c l}(\eta) \frac{\langle\tilde{\psi}(\eta)|\hat{K}| \tilde{\psi}(\eta)\rangle}{\left\langle\tilde{\psi}(\eta)\left|\hat{K}^{2}\right| \tilde{\psi}(\eta)\right\rangle} \\
& =2 \alpha K_{c l} \frac{\sqrt{\pi} \alpha K_{c l} \operatorname{Erfc}\left(-\alpha K_{c l}\right)+e^{-\alpha^{2} K_{c l}^{2}}}{\sqrt{\pi}\left(1+2 \alpha^{2} K_{c l}^{2}\right) \operatorname{Erfc}\left(-\alpha K_{c l}\right)+2 \alpha K_{c l} e^{-\alpha^{2} K_{c l}^{2}}}
\end{aligned}
$$

It is now convenient to change the variable of integration from the conformal time to the Schwarzschild time according to Eq. (2.12) and define:

$$
\left\{\begin{array}{l}
\Delta t \equiv t^{\prime \prime}-t^{\prime} \\
T \equiv\left(t^{\prime \prime}+t^{\prime}\right) / 2 .
\end{array}\right.
$$

Eq. (4.3), with $\eta^{\prime \prime}\left(t^{\prime \prime}=L\right)=\eta^{\prime}\left(t^{\prime}=L\right)=\bar{\eta}$ and $\eta^{\prime \prime}\left(t^{\prime \prime}=0\right)=\eta^{\prime}\left(t^{\prime}=0\right)=\eta_{0}$, then becomes:

$$
\begin{aligned}
P(\omega, L) \simeq & \frac{Q_{\omega}{ }^{2}}{\hbar^{2}} \int_{0}^{L} d T \frac{C_{\epsilon}{ }^{2} e^{-\frac{T}{M}}}{4 M^{2}} \int_{2|T-L / 2|-L}^{L-2|T-L / 2|} d(\Delta t) e^{i \omega \Delta t} \\
& \times R(T+\Delta t / 2) R(T-\Delta t / 2) D_{\epsilon}(\Delta t, T) .
\end{aligned}
$$

We now note that $D_{\epsilon}$ is singular for:

$$
\begin{array}{ll}
\cos \left(\eta^{\prime \prime}-\eta^{\prime}-i \varepsilon\right)=1 & \epsilon=+1 \\
\left(\eta^{\prime \prime}-\eta^{\prime}-i \varepsilon\right)^{2}=0 & \epsilon=0,-1 .
\end{array}
$$

Using Eq. (2.13) one has near the horizon:

$$
\eta^{\prime \prime}-\eta^{\prime} \simeq C_{\epsilon} e^{-\frac{t^{\prime \prime}}{2 M}}-C_{\epsilon} e^{-\frac{t^{\prime}}{2 M}}=-2 C_{\epsilon} e^{-\frac{T}{2 M}} \sinh \left(\frac{\Delta t}{4 M}\right)
$$


hence on substituting into Eqs. (4.10) in the limit $\varepsilon \rightarrow 0$, one finds double poles at:

$$
\Delta t_{q}=4 \pi i q M
$$

where $q$ is an integer or zero. Apart from these singularities the integrand in Eq. (4.9) is an analytic function in the complex variable $\Delta t$. Thus one can close the integration path with a semicircle of radius $L-2|T-L / 2|$ in the upper half complex $\Delta t$ plane and apply the theorem of residues. In the limit for $L \rightarrow \infty$ the integral along the semicircle vanishes due to the energy exponential and the possible contributions come from the residues alone.

Let us further examine the above singularities. In terms of the conformal time near the horizon one finds:

$$
\begin{aligned}
& \eta^{\prime \prime}-\eta_{H}=(-1)^{q} C_{\epsilon} e^{-\frac{T}{2 M}} \\
& \eta^{\prime}-\eta_{H}=(-1)^{q} C_{\epsilon} e^{-\frac{T}{2 M}} .
\end{aligned}
$$

It is clear that Eqs. (4.13) have the opposite sign to the one in Eq. (2.13) for $q=2 n+1$ and therefore we must consider only the residues at even $q$, that is:

$$
\Delta t_{2 n}=8 \pi i n M
$$

which are the poles that are associated with a free fall from outside the horizon. The ones with odd $q$ correspond to geodesic motion leaving the horizon from inside. Thus for an observer outside the horizon the only relevant poles are the ones given in Eq. (4.14).

The residues to be summed over then are:

$$
\begin{aligned}
S_{n} & =\lim _{\Delta t \rightarrow \Delta t_{2 n}} \frac{d}{d(\Delta t)}\left[\left(\Delta t-\Delta t_{2 n}\right)^{2} e^{i \omega \Delta t} \bar{D}_{\epsilon}(\Delta t, T)\right] \\
& =-\frac{4 M^{2} \hbar}{C_{\epsilon}{ }^{2}} e^{\frac{T}{M}} \frac{d}{d(\Delta t)}\left[R(T+\Delta t / 2) R(T-\Delta t / 2) e^{i \omega \Delta t}\right]_{\Delta t=\Delta t_{2 n}},
\end{aligned}
$$

where $R$ is given in terms of $\eta$ in Eq. (4.7). Further:

$$
\left.\frac{d}{d(\Delta t)} R(T+\Delta t / 2)\right|_{\Delta t=\Delta t_{2 n}}=-\left.\frac{d}{d(\Delta t)} R(T-\Delta t / 2)\right|_{\Delta t=\Delta t_{2 n}}
$$

so that the expression Eq. (4.15) simplifies to:

$$
S_{n}=-\frac{4 M^{2} \hbar}{C_{\epsilon}^{2}} e^{\frac{T}{M}} R^{2}(T) i \omega e^{-\beta \hbar \omega n},
$$

with $\beta \equiv(8 \pi M) / \hbar$.

After performing the $\Delta t$ integration the probability amplitude becomes:

$$
P(\omega, L) \simeq \frac{Q_{\omega}^{2} \omega}{2 \pi \hbar} \int_{0}^{L} d T R^{2}(T) \sum_{n=0}^{N} e^{-\beta \hbar \omega n} .
$$


where $N$ is the largest integer $\leq(L-2|T-L / 2|) / 8 \pi M$ and we have omitted the integral along the contour which does not contribute for $L$ large. We observe that in order to include at least one pole inside the contour of integration $L$ must be greater than $\left|\Delta t_{2}\right|=$ $8 \pi M$, which implies:

$$
\eta-\eta_{H} \simeq C_{\epsilon} e^{-\frac{T}{2 M}}<C_{\epsilon} e^{-2 \pi}
$$

and if we consider the shell very close to the horizon, that is values of $T$ large in Eqs. (4.18) and (4.19), we obtain a probability amplitude per unit time equal to:

$$
\frac{P(\omega, L)}{L} \simeq \frac{Q_{\omega}{ }^{2} R^{2}(\infty)}{2 \pi \hbar} \frac{\omega}{1-e^{-\beta \hbar \omega}},
$$

which is a Planck distribution with the usual Hawking temperature $T_{H}=1 / \beta$ and $R(\infty)$ is a non zero constant given by $R\left(\eta_{H}\right)$. Let us note that the terms omitted in the approximations that lead to Eq. (4.20) are mainly concerned with effects at small time $T$ : in fact we let $L$ go to infinity and neglected factors of $e^{-T / 2 M}$. The corrections one expects when relaxing these assumptions are relevant for small values of $T$ and should lead to transient effects when the monopole starts radiating or the horizon is forming.

In the classical point-like limit, Eq. (3.36), $R \rightarrow 1$ and one recovers the usual field theory in the fixed Robertson-Walker-like background. In this last case Eq. (4.20) in the large $L$ limit becomes:

$$
\frac{P(\omega, L)}{L} \simeq \frac{Q_{\omega}^{2}}{2 \pi \hbar} \frac{\omega}{1-e^{-\beta \hbar \omega}}
$$

which is equal to Eq. (4.20) apart from the factor of $R^{2}(\infty)$. If we However take the alternative limit, Eq. (3.37), that is $b \rightarrow 0$, one finds that the dust shell decouples from the conformal field, since:

$$
R \sim \alpha \rightarrow 0
$$

and there is no emission:

$$
P(\omega, L) \rightarrow 0
$$

One may speculate that this effect can be used to eliminate ultra-planckian effects. In fact, it is known that, if $\omega$ is the frequency of the emitted quanta as is measured by a distant observer, a fixed observer located near the point of emission at $r=r_{0}$ will measure instead a blue-shifted frequency:

$$
\omega^{*}=\left(1-\frac{2 M}{r_{0}}\right)^{-1 / 2} \omega
$$


and this expression clearly gives $\omega^{*}>m_{p} / \hbar=\ell_{p}{ }^{-1}$ for $r_{0}$ sufficiently close to $r_{H}$. In order to probe these modes, one must use an emitter (detector) localized in a region smaller than $\omega^{-1} \sim \ell_{p}$. So one expects that $b$ in the wave function Eq. (3.32) should be less than $\ell_{p}$ for our collapsing shell to couple with conformal quanta of ultra-planckian energies. But this would correspond to the limit in Eq. (3.37) which in turn implies Eq. (4.23): ultra-planckian energy quanta are not emitted by the monopole.

A further point worth noting is that $R(\infty)$ given by $R\left(\eta=\eta_{H}\right)$ in Eq. (4.7) actually depends on $\epsilon$ through $\alpha$ (see Eq. (3.33)) but not through $K_{c l}\left(\eta_{H}\right)=2 M / \rho_{0}$. This of course implies that the probability amplitude in Eq. (4.20) depends on the internal dust sphere metric. Indeed since:

$$
\alpha\left(\eta_{H}\right)=\frac{b}{\left[4 \ell_{p}^{4} \rho_{0}^{2}\left(1-\epsilon \rho_{0}^{2}\right)+b^{4}\left(1-2 \epsilon \rho_{0}^{2}\right)^{2}\right]^{1 / 2}},
$$

one would have in the limit $b \gg \ell_{p}, \alpha\left(\eta_{H}\right) K_{c l}\left(\eta_{H}\right) \rightarrow 0$ :

$$
R\left(\eta_{H}\right) \simeq \frac{2}{\sqrt{\pi}} \alpha\left(\eta_{H}\right) K_{c l}\left(\eta_{H}\right) \simeq \begin{cases}\frac{4 M}{\sqrt{\pi}} \frac{1}{b \rho_{0}\left(2 \rho_{0}^{2}-1\right)} & \epsilon=+1 \\ \frac{4 M}{\sqrt{\pi}} \frac{1}{b \rho_{0}} & \epsilon=0 \\ \frac{4 M}{\sqrt{\pi}} \frac{1}{b \rho_{0}\left(2 \rho_{0}^{2}+1\right)} & \epsilon=-1,\end{cases}
$$

that is one has different emission rates depending on the internal geometry of the sphere of dust.

Let us end with a speculation: we considered the matching condition in the classical limit, if one wished to consider quantum mechanical corrections one should replace it by the continuity of the gravitational wave function inside the dust (Robertson-Walker) with that outside (Schwarzschild). This suggests the results in Eq. (4.26) should not change dramatically immediately outside the dust, implying a form of quantum hair leading to information on the geometry in the black hole through the intensity of the radiation.

\section{Conclusions}

We have studied the collapse of a self-gravitating homogeneous sphere of dust in the context of canonical general relativity by applying the Born-Oppenheimer approach to the coupled dust-gravity system. Such an approach allows for the quantization both 
of matter and gravity and it is only in the semiclassical limit for the latter and in the absence of fluctuations that time emerges and one recovers quantum theory for matter on a classical background. This has allowed us, in some measure, to estimate quantum mechanical effects both for matter and gravity.

The gravitational wave packets obtained correspond to the quantum mechanical evolution of a gaussian packet of initial width $b\left(=\ell_{p}\right.$ for a minimum wave packet $)$ centred on the classical trajectory $K_{c l}$. This has allowed us to examine the conditions for which one recovers the usual "point-like" classical collapse which, for example, was necessary to implement the matching (or junction) condition.

Further the fact matter (dust) is also quantised has allowed us to also estimate matter fluctuations which lead to a violation of the adiabatic approximation. The validity of the approximation not surprisingly requires that the Schwarzschild radius of the dust sphere be greater than the Compton wavelength of the matter (dust) particles, indeed should this not be the case one would naturally expect fluctuations corresponding to the creation of particles by the gravitational field to be important.

One of the most interesting quantum effects in black hole physics is Hawking radiation and in order to to study this we considered a static monopole, with an internal energy spectrum, situated near the horizon which is being formed in coincidence with the collapse of the last shell. The monopole couples gravitation to a conformal scalar field which has support both inside and outside the dust sphere. We found that a thermal Wightman's function corresponding to a Planckian distribution with the Hawking temperature appeared in the de-excitation (or excitation) probability of the monopole.

Again the fact that our last dust shell is described by a wave function allowed us to discuss various limits and differing orders of limits. Let us just mention that although one always had a thermal Hawking temperature spectrum, the intensity of the radiation depended on the internal dust metric. Should the result also be true just outside the dust shell (a sort of quantum mechanical "matching" condition) one would have a correction to the classical no-hair theorem.

The most serious drawback in our approach to Hawking radiation is the neglect of back-reaction effects. In fact, one would expect the emitted quanta to alter the metric outside the dust (since we no longer have vacuum) and also inside, since the outer shell loses mass. We hope to return to this and other points.

\section{Acknowledgements}


We wish to thank R. Balbinot, R. Bergamini and R. Brout for many helpful discussions and suggestions.

\section{A Useful integrals}

In the text we defined:

$$
\left\langle\hat{K}^{n}\right\rangle \equiv \frac{\left\langle\tilde{\psi}\left|\hat{K}^{n+2}\right| \tilde{\psi}\right\rangle}{\left\langle\tilde{\psi}\left|\hat{K}^{2}\right| \tilde{\psi}\right\rangle},
$$

where $\left\langle\tilde{\psi}\left|\hat{K}^{2}\right| \tilde{\psi}\right\rangle$ is the norm of the wave function $K \tilde{\psi}$. If one further defines the following differential operator:

$$
D \equiv \frac{1}{2 \alpha^{2}} \frac{d}{d K_{c l}}+K_{c l}
$$

then one has:

$$
\begin{aligned}
\left\langle\tilde{\psi}\left|\hat{K}^{n}\right| \tilde{\psi}\right\rangle & =D^{n} \int_{0}^{\infty} d K e^{-\alpha^{2}\left(K-K_{c l}\right)^{2}} \\
& =D^{n}\left[\frac{\sqrt{\pi}}{2 \alpha} \operatorname{Erfc}\left(-\alpha K_{c l}\right)\right] .
\end{aligned}
$$

In particular one finds:

$$
\begin{aligned}
& \langle\tilde{\psi}|\hat{K}| \tilde{\psi}\rangle=\frac{1}{2 \alpha^{2}}\left[\sqrt{\pi} \alpha K_{c l} \operatorname{Erfc}\left(-\alpha K_{c l}\right)+e^{-\alpha^{2} K_{c l}^{2}}\right] \\
& \left\langle\tilde{\psi}\left|\hat{K}^{2}\right| \tilde{\psi}\right\rangle=\frac{1}{4 \alpha^{3}}\left[\sqrt{\pi}\left(1+2 \alpha^{2} K_{c l}^{2}\right) \operatorname{Erfc}\left(-\alpha K_{c l}\right)+2 \alpha K_{c l} e^{-\alpha^{2} K_{c l}^{2}}\right] \\
& \left\langle\tilde{\psi}\left|\hat{K}^{3}\right| \tilde{\psi}\right\rangle=\frac{1}{4 \alpha^{4}}\left[\sqrt{\pi} \alpha K_{c l}\left(3+2 \alpha^{2}{K_{c l}}^{2}\right) \operatorname{Erfc}\left(-\alpha K_{c l}\right)\right. \\
& \left.+2\left(1+\alpha^{2} K_{c l}^{2}\right) e^{-\alpha^{2} K_{c l}^{2}}\right] \\
& \left\langle\tilde{\psi}\left|\hat{K}^{4}\right| \tilde{\psi}\right\rangle=\frac{1}{8 \alpha^{5}}\left[\sqrt{\pi}\left(3+12 \alpha^{2} K_{c l}^{2}+4 \alpha^{4} K_{c l}^{4}\right) \operatorname{Erfc}\left(-\alpha K_{c l}\right)\right. \\
& \left.+2 \alpha K_{c l}\left(5+2 \alpha^{2} K_{c l}^{2}\right) e^{-\alpha^{2} K_{c l}^{2}}\right] \text {. }
\end{aligned}
$$

\section{B Evaluation of the Fluctuations}

From the definition Eq. (3.10) one has:

$$
\left\langle\tilde{\chi}_{N}\left|\frac{\partial^{2}}{\partial K^{2}}\right| \tilde{\chi}_{N}\right\rangle=\left\langle\chi_{N}\left|\left(\frac{\partial}{\partial K}-\left\langle\frac{\partial}{\partial K}\right\rangle\right)\left(\frac{\partial}{\partial K}-\left\langle\frac{\partial}{\partial K}\right\rangle\right)\right| \chi_{N}\right\rangle
$$




$$
=\sum_{P \neq N}\left\langle\chi_{N}\left|\frac{\partial}{\partial K}\right| \chi_{P}\right\rangle\left\langle\chi_{P}\left|\frac{\partial}{\partial K}\right| \chi_{N}\right\rangle .
$$

From the identity:

$$
\frac{\partial}{\partial K}\left(\hat{H}_{M}\left|\chi_{N}\right\rangle\right)=\frac{\partial \hat{H}_{M}}{\partial K}\left|\chi_{N}\right\rangle+\hat{H}_{M} \frac{\partial}{\partial K}\left|\chi_{N}\right\rangle
$$

on contracting over $\left\langle\chi_{L}\right|$ for $L \neq N$ one obtains:

$$
\left\langle\chi_{L}\left|\frac{\partial}{\partial K}\right| \chi_{L}\right\rangle=\frac{\left\langle\chi_{L}\left|\frac{\partial \hat{H}_{M}}{\partial K}\right| \chi_{N}\right\rangle}{E_{N}-E_{L}},
$$

where one has $\hat{H}_{M}\left|\chi_{N}\right\rangle=E_{N}\left|\chi_{N}\right\rangle=\hbar \mu(N+1 / 2)\left|\chi_{N}\right\rangle$. Further:

$$
\begin{aligned}
\frac{\partial \hat{H}_{M}}{\partial K} & =-\frac{3}{2}\left[\frac{\hat{\pi}_{\phi}^{2}}{K^{4}}+\mu^{2} K^{3} \phi^{2}\right] \\
& =\frac{3}{2} \frac{\hbar \mu}{K}\left(\left(\hat{a}^{\dagger}\right)^{2}+\hat{a}^{2}\right),
\end{aligned}
$$

where $\hat{a}^{\dagger}$ and $\hat{a}$ are the creation and annihilation operators for the scalar field $\hat{\phi}$ :

$$
\begin{gathered}
\hat{\phi}=\sqrt{\frac{\hbar}{2 \mu K^{3}}}\left(\hat{a}^{\dagger}+\hat{a}\right) \\
\hat{\pi}_{\phi}=i \sqrt{\frac{\hbar \mu K^{3}}{2}}\left(\hat{a}^{\dagger}-\hat{a}\right) .
\end{gathered}
$$

On substituting into Eq. (B.1) one finally has:

$$
\begin{aligned}
\left\langle\tilde{\chi}_{N}\left|\frac{\partial^{2}}{\partial K^{2}}\right| \tilde{\chi}_{N}\right\rangle & =-\sum_{P \neq N} \frac{1}{\left(E_{N}-E_{P}\right)^{2}}\left\langle\chi_{N}\left|\frac{\partial \hat{H}_{M}}{\partial K}\right| \chi_{P}\right\rangle\left\langle\chi_{P}\left|\frac{\partial \hat{H}_{M}}{\partial K}\right| \chi_{N}\right\rangle \\
& =-\frac{9 \hbar^{2} \mu^{2}}{4 K^{2}} \frac{(N+1)(N+2)}{\left(E_{N}-E_{N+2}\right)^{2}}-\frac{9 \hbar^{2} \mu^{2}}{4 K^{2}} \frac{N(N-1)}{\left(E_{N}-E_{N-2}\right)^{2}} \\
& =-\frac{9}{8 K^{2}}\left(N^{2}+N+1\right) .
\end{aligned}
$$

\section{References}

[1] DeWitt B S 1967 Phys. Rev. 1601113

[2] Arnowitt R Deser S and Misner C 1962 in Gravitation: An Introduction to Current Research edited by Witten L. (New York: Wiley)

[3] Banks T 1985 Nucl. Phys. B249 332; Brout R 1987 Found. Phys. 17603 and 1987 Z. Phys. B 60 359; Brout R and Venturi G 1989 Phys. Rev. D 152436 
[4] Hawking S W 1974 Nature (London) 248 30; 1975 Comm. Math. Phys. 43199

[5] Madsen M S 1988 Class. Quantum Grav. 5627

[6] Lund F 1973 Phys. Rev. D 83253

[7] Tolman R C 1934 Proc. Nat. Acad. Sci. 20169

[8] Oppenheimer J R and Snyder H 1939 Phys. Rev. 56455

[9] Berger B K Chitre D M Moncrief V E and Nutku Y 1972 Phys. Rev. D 52467

[10] Misner C W Thorne K S and Wheeler J A 1973 Gravitation (San Francesco: Freeman)

[11] Israel W 1966 Nuovo Cimento 44 B 1

[12] Venturi G 1992 Class. Quantum Grav. 91217

[13] Feynman R P and Hibbs A R 1965 Quantum Mechanics and Path Integrals (New York: McGraw-Hill)

[14] Gibbons G W and Hawking S W 1977 Phys. Rev. D 152752

[15] Bekenstein J D 1974 Lett. Nuovo Cimento 11476

[16] Peleg Y 1995 Phys. Lett. B 356462

[17] For a general discussion of Ehrenfest's theorem in quantum gravity see: Greensite J 1991 Nucl. Phys. B 351749

[18] Birrell N D and Davies P C W 1982 Quantum Fields in Curved Space (Cambridge Univ. Press)

[19] Unruh W G 1976 Phys. Rev. D 14870

[20] Casadio R and Venturi G 1995 Phys. Lett. A 19933 